\title{
Ueber einen Fall von psoriasiformem und lichenoidem Exanthem.
}

Von

Dr. Fritz Juliusberg,

Assistenzarzt.

Am 16. Mai 1895 wurde auf die dermatologische $A b$ theilung des städtischen Krankenhauses zu Frankfurt a. M. ein Patient mit einer Hautaffection aufgenommen, die ibrer Eigenart wegen einiges Interesse verdient.

Der Patient, Sp., Geschäftsreisender, 23 Jahre alt, stammt aus einer Familie, bei der in keiner Hinsicht eine Vererbung pathologischer Zustände nachzuweisen ist Dem Patienten selbst ist es aufgefallen, dass er bei gewissen äusseren Reizen, z. B. bei der Einwirkung der Wärme, bei der Wirkung der Sonnenstrahlen, nach stärłeren Anstrengungen, sowie beim Angesprochen- und Angesehenwerden stark und schnell erröthete. Das Erröthen war an den betroffenen Stellen mit heftigem Jucken verbunden. Auch traten häufig Juckanfälle beim Genuss warmer Speisen und zwar am Rücken auf. Allen heftigen Juckanfällen ging stets Röthung der Haut voraus. Im Alter von 17 Jahren bemerkte der Patient zuerst Schuppen and rothe Fleckchen arn Körper, zuerst an den Oberarmen, dann an den Beinen. An Brust und Bauch ist dem Patienten nie eine reichliche Schuppung aufgefallen, dagegen sah er auch hier rothe Fleckchen. Patient hat wegen des Juckens auf ärztlichen Rath das Baden seit 3 Jahren gänzlich ausgesetzt und glaubt hierauf die Scbuppenbildung zurückführen zu müsı̈en.

Soviel zur Anamnese des Falles, dessen Status ich jetzt folgen lasse.

Beim Anblicken und Anreden des Patienten tritt ein Erythema pudoris faciei auf. Ferner lässt sich eine Urticaria factitia hervorrufen.

Die Haut des Patienten ist an den gesunden Partien trocken und blass. Die erkrankten Hantpartien lassen sich am besten getrennt nach den einzelnen Körperregionen beschreiben.

Auf der blassen Halspartie, an welcher die Halsvenen bläulich durchschimmern, fnden sich scharf abgegrenzte Fleckchen and weiche Papeln von meist runder, seiten ovaler Form und von rothgelber, fast 
orangegelber Farbe. Die Randzone der maculösen Efflorescenzen, die normale Hautfältelung zeigen, ist orangegelb; die Mittelpartie ist eingesunken, sie scheint atrophisch, ibre Färbung ist gelbbraun. Die Papeln sind kräftiger gefärbt wie die Flecken und zeigen normale Hautfältelung. Zugleich bestehen am Hals vorn an 6 Stellen hirsen- bis knapp linsengrosse dünne und silberglänzende Schüppchen in normaler Umgebung. Am oberen Drittel des Halses bis zu den Ohren reichend sieht man zahlreiche, wie Epheliden aussehende, stecknadelkopfgrosse hellbraune Pigmentationen. Brustpartie: Auf der Vorderseite beider Schultern befinden sich zahlreiche knapp linsengrosse zarte und glänzende Schüppchen, die sich peripher, wo sie fast durchsichtig sind, leicht von der Unterlage abheben lassen. Central haften sie fest an der Unterlage; entfernt man sie, so erscheinen vereinzelte feinste Bluttröpfchen. Oberhalb der rechten Mammilla besteht ein zehnpfennigstückgrosser Ring, der eine normale Hautzone umgibt. Der Ring wird gebildet von aneinandergereihten bräunlichen zarten Schüppchen, die sich um die Follikel anordnen. Letztere treten als schwarze Pünktchen deutlich hervor.

Die Seitenflächen des Bauches sind mit bis kleinfingergrossen Schüppchen bedeckt, welche nach den Beinen zu an Grösse und Dichtigkeit zunehmen und in der Trochanterhöhe schon zusammenhängende Lamellen bilden. Die Schüppchen verhalten sich ähnlich, wie die an der Brust beschriebenen.

Am Rücken finden sich keine schuppenden Stellen, dagegen die am Halse beschriebenen orangerothen Papeln in grosser Anzahl. Viele lassen eine deutliche Atrophie im Centrum erkennen.

Beide Schultern zeigen neben normalen Hautstellen eine scheinbar diffuse Schuppung. Doch lässt sich auch hier feststellen, dass innerhalb der unregelmässigen Schuppungspartien einzelne oft dicht aneinandergereihte erbsengrosse Schuppen auftreten, mit ziemlich scharfer Begrenzung, leicht bräunlìch gefärbt, gegenüber dem Silberglanz der diffus verbreiteten Schüppchen. Die Schüppchen haften mit dem Centrum fest an der Unterlage. Nach ihrer Entfernung tritt keine capilläre Blutung ein, sondern man hat die normale Hautdecise vor sich.

Der Oberarm ist mit zahlreichen erbsengrossen Schuppen und grösseren fingernagelgrossen Schuppenlamellen bedeckt. Diese lassen sich ziemlich schwer von der Unterlage abschaben. Die darunterliegende Haut ist hellglänzend, sonst ohne Besonderheiten. Dasselbe Bild liefern die Schuppen auf den Impfnarben. Streck- und Bengeseite ist gleichmässig betheiligt. An den nicht schuppenden Stellen ist die Haut trocken glänzend, gelblich verfärbt. Die Fältelung der Haut ist deutlicher ausgesprochen, als bei der normalen Haut; sie bildet ein grobes Netzwerk. Nach dem Ellenbogen zu wird die Fältelung zarter. Daselbst ist die Färbung weissglänzend. Dort finden sich mehrfach leicht gebräunte linsengrosse Fleckchen ohne Schuppen. Zugleich finden sich an beiden Oberarmen versprenkelte orangegelbliche Fleckehen, wie sie am Halse beschrieben sind. Die Oberschenkel zeigen an der Hinter, Vorder- 
und) Aussenseite dieselben Verhältnisse, wie am Bauch; die Innenseite ist völlig frei.

Ganz andere Verhältnisse zeigt die Haut abwärts von den Knien. Hier überwiegt die Atrophie vollständig. Die Haut ist sehr blass, dünn und trocken. Beim Darüberstreichen fliegen ganz feine Schüppchen in grossen Massen auf. Die oben beschriebenen peripher haftenden Schüppcleen sind besonders in der Kniegegend nagelgross und dicht aneinandergereiht, so dass die normale Hautfältelung nicht mehr zu erkennen ist. Distalwärts vereinigen sich bisweilen die Schuppen zu grösseren Lamellen, meist aber sind es wieder, wie am Arme, dicht beisammen sitzende Schüppchen ohne Röthung oder sonstige Veränderung der Umgebung. Nach Abkratzung der Schüppchen besteht hier überall blasse, atrophische aber nicht erodirie Epitheldecke. Die dicht gereibten Schüppchen und die trockene Haut machen hier den Eindruck der Ichthyosis.

Die Soblen zeigen lamellöse Schuppen. Die Haut fühlt sich normal dick an und hat den gewöhnlichen weiss-gelblichen Ton. Die lamellösen Schuppen finden sich links an der Ferse, rechts auf der ganzen. lateralen Sohlenbälfte. Die Schuppen liegen hier fast flach, dachziegelartig übereinander und so fühlt sich die Haut beim Darüberstreichen an, wie ein Reibeisen. Die Nägel sind überall frei.

Der Kopf zeigt, soweit die Behaarung reicht, diffuse Schuppung ohne Besonderheiten, also das Bild der Seborrhoea sicca.

Innerhalb der nächsten zwei Jahre stellte sich der Patient von Zeit zu Zeit vor und wir konnten constatiren, dass in dieser Zeit die Papeln, Flecken und schuppenden Stellen im Allgemeinen dieselben blieben, wenn auch Exacerbationen und Remissionen des Leidens auftraten und zeitweise das Krankheitsbild etwas veränderten. Während die Angioneurose in dieser Zeit dieselbe blieb, hatten wir Gelegenheit zu bemerken, dass die einzelnen Efflorescenzen mit der Entstehung einer kleinen etwa stecknadelkopfgrossen, hochrothen Papel beganmen. Diese Papel verbreiterte sich und warde dabei flacher, wobei das intensive Rotb immer mehr zu einem matten Rosa abblasste, bis schliesslich an der Stelle der ursprünglichen Papel eine fünfpfennigstückgrosse Macula sich vorfand. Diese Maculae verschwanden bald später, bald früher und neue Efflorescenzen, die die obigen Metamorphosen durchmachten, traten an ihre Stelle. Erst jetzt, 2 Jahre nach der Anlage der obigen Krankengeschichte, traten Efflorescenzen von einem ganz anderen Charakter und zwar am Halse auf. Dort erschienen nämlich auf der linken Seite in der Höhe der vertebra prominens 2 stecknadelkopfgrosse Knötchen von wächsernem Glanz, die eine kleine Delle in der Mitte trugen und 5 weitere Knötchen von gleicher Farbe und. Grösse, an deren Spitze eine kleine Schuppe aufgelagert war.

Die mikrosk o p is che Untersuchung eines vor 2 Jahren am linken Vorderarm und zwar an einer schuppenden Stelle excidirten Hautstuickchens ergab folgenden Befund: 
Das Stratum corneum weist eine erhebliche Verdickung auf. Diese beruht auf einer Vermehrung seiner Lamellen, die in den unteren Partien dicht aneinandergefügt sind, in den oberen Schichten dagegen ganz aufgelockert erscheinen. Die lamellösen Auflagerungen folgen den Wellenlinien der Papillarschicht und füllen die Vertiefungen derselben aus. Die stärkste Hyperkeratose findet sich an der Mïndung der Follikel. Am Haarschaft selbst und an der Haarwurzelscheide ist nichts besonders wahrzunehmen, abgesehen davon, dass eine noch normale Kernretraction namentlich in den unteren Partien, aber auch in den mittleren vorkommt.

Diese Retractionsfiguren kommen in dem Stratum granulosum der Epidermis regelmässig vor und steigern sich bis zum vollständigen Verschwinden des Kernes. Die Malpighische Schicht zeigt weder eine Dickenzunahme noch weichen ihre Zellen von der Norm ab, aber die interpapillären Retezapfen sind an einigen Stellen weit ausgezogen. Eine Veränderung der Malpigbischen Sehicht, etwa eine erheblichere kleinzellige Infiltration ist nicht wahrzunehmen, nur an einzelnen Stellen ist eine geringe Rundzellen-Einwanderung vorhanden.

Die Papillen sind sämmtlich kleinzellig infiltrirt. Diese Infiltration ist nicht in allen Papillen eine gleichmässige, sondern sie ist fleckweise vertheilt. Auch in den an die Papillen angrenzenden Schichten des Coriums ist die kleinzellige Infiltration eine erhebliche, während die tieferen Schichten von Rundzelleninfiltraten frei sind. Diese Infiltrate, in meist streifiger Form auf den Schnitten, liegen zum grösseren Theile regellos, zum geringeren Theile folgen sie dem Verlaufe der Gefässe, deren Wand sie ringförmig umgeben. Die kleinzellige Infiltration dringt sogar in die Adrentitia und Media der Arterien ein. Die Drüsen der Haut zeigen gar keine abnormen Verhältnisse. Das Netz der elastischen Fasern, nach Unna-Tänzers Orceinmethode dargestellt, ist gut ausgebildet, auch die in die infiltrirten Papillen steigenden Schlingen. Im Corium werden die elastischen Fasern durch die Infiltrate an einzelnen Stellen auseinandergedrängt; in den tieferen Schichten des Coriums sind die elastischen Fasern erheblich verdickt und bilden theilweise breite Lamellen. 
Ueb. einen Fall v. psoriasiformem u. Tichenoidem Exanthem. 261

Es erhebt sich nun die Frage, ob das beschriebene Krankheitsbild in so engen Beziehungen zu einer der bekannten Gruppen von Hautkrankheiten steht, dass man es in diese zwanglos einreihen kann, oder ob es eine neue eigenartige Erkrankung darstellt.

Stellen wir zunächst einen Vergleich mit dem Eczema chronicum an, zu dem auch die Polymorphie unserer Efflorescenzen uns auffordert. Zunächst ist mit dieser Hautkrankheit der Begriff des Juckens unzertrennlich verbunden. Dieses Symptom trat in unserem Falle gar nicht oder ganz unbedeutend auf. Zweitens hat das Eczem, wenn es einmal in solchem Masse auftritt, die Tendenz, sich flächenhaft auszubreiten und sich nicht in streng separirten Einzelefflorescenzen zu markiren. Drittens pflegt ein Eczem bei längerer Daver eine Reihe verschiedener Stadien durchzumachen, worunter man das Stadium des Nässens selten vermissen wird. Auch dieses konnten wir in unserem Falle nicht beobachten. Ziehen wir differentialdiagnostisch in zweiter Linie das s. g. Fezem en plaque in Betracht, so ergeben sich auch hier ganz wesentliche Unterschiede. Beim Eezem en plaque treten die ersten Efflorescenzen nur am Stamm auf, erst beim Weiterschreiten geht es von da weiter auf die Extremitäten über. In unserem Falle hat die Krankheit gerade den umgekehrten Weg eingeschlagen und ist zuerst an den Extremitäten aufgetreten. Ferner kommt ( $\mathrm{K}$ a p o s i) ${ }^{\mathbf{1}}$ ) das Eezem en plaque fast nur bei anämischen, schwachen Individuen - mit Vorliebe bei Weibern - vor.

Auch der mikroskopische Befund spricht gegen diese Diagnose: Wir hatten Gelegenbeit, einen typischen Fall von Eczem en plaque klinisch und auch mikroskopisch zu untersuchen. In den excidirten Hautstïckchen fehlte gänzlich die starke Hypertrophie der Hornschicht, die im Falle Sp. so sehr in den Vordergrund tritt. Eine Verdickung der Malpighischen Schicht war deutlich zu erkennen. Im Corium fanden sich zahlreiche umschriebene Infiltrate, die besonders, wie im Falle Sp., in den Papillen sich localisirten. Doch war bei diesem Eczem en plaque die Infiltration eine viel erheblichere. Besonders

1) Kaposi, Verhandlungen der Wiener dermatologischen Gesellschaft. Referat: Arohiv für Dermatologie und Syphilis. 1896. 
auffallend war aber eine Erkrankung der Gefässe und zwar eine Endarteriitis intimae, die soweit ging, dass eine Reihe von Gefässen thrombosirt waren.

Noch leichter lässt sich unser Fall Sp. differentialdiaguostisch ron der Psoriasis trennen, obschon auf den ersten Blick einige Aehnlichkeiten mit dieser Hautkrankheit auffallen: dies sind die Umschriebenheit der Efflorescenzen und die Schuppung auf denselben. Dagegen fehlte unserem Falle die für Psoriasis als charakteristisch angenommene Localisation auf den Streckseiten der Gelenke, die bei einer so ausgedehnten Affection, wie es unsere ist, meist deutlich hervortritt. Aber auch von dieser Regel finden sich genügend Ausnahmen, so dass man diesen Grund allein nicht als stichhaltig gegen die Diagnose Psoriasis ins Feld führen kann. Dagegen bietet die Betrachtung der einzelnen Efflorescenzen ror allem makroskopisch, weniger mikroskopisch ein von Psoriasis durchaus verschiedenes Bild.

In erster Linie ist die Farbe der psoriatischen Efflorescenz eine rothe bis rothbraune, nie eine orangegelbe.

Zweitens, sei es dass man den psoriatischen Plaque als Papel oder als mit Schuppe überdeckte Macula auffasst, immer wird der tastende Finger beim Darüberstreichen einem rauhen Widerstand begegnen und nach Entfernen der Schuppe wird eine capilläre Blutung nicht ausbleiben. In unserem Falle treten neben sehr weichen Papeln ganz besonders Flecken in den Vordergrund des Krankheitsbildes, die nur dem Auge, nicht dem Tastgefühl sich kund thun.

Die Schuppung ist, trotzdem der Patient auf ärztlichen Rath das regelmässige Baden schon längere Zeit ausgesetzt hat, eine sehr geringe, was sich daraus erklärt, dass die Schuppung in unserem Falle nur ein Endstadium ist; eine capilläre Blutung nach Abkratzen der Schuppen tritt sebr unregelmässig und nur in geringem Masse auf.

Mikroskopisch fällt bei unserem Fall manche Aehnlichkeit mit dem Bilde der Psoriasis auf, doch ist vor allem die Infiltration des Coriums beim Falle Sp. eine viel geringere, als es bei einer lange dauernden Psoriasis zu sein pflegt. Im Verhalten der Epidermis bietet sich differentialdiagnostisch nichts. 
Ueb. einen Fail v. psoriasiformem u. lichenoidem Exanthem. 263

von Psoriasis abweichendes. Trotz alledem genügen aber die makroskopischen Differenzen, unsere Krankheit als ganz verschieden selbst von einer atypischen Psoriasis aufzufassen.

Es eriibrigt sich, auf eine eingehendere Vergleichung mit der Pityriasis rubra (Hebrae) einzugehen, da bei dieser Krankheit keine anderen Symptome als Röthung und Schuppung auftreten. Derselbe Gesichtspunkt veranlasst uns, auf eine Nebeneinanderstellung mit der Pityriasis rosée (Gibert) zu verzichten.

Gegen Herpes tonsurans maculosus et squamosus spricht auf den ersten Blick die Farbe unserer Papel, das theilweise zusammenfliessen unserer Efflorescenzen, aber noch schwerwiegender die Resistenz gegen Chrysurobin, das 2 Monate in unserem Falle angewandt wurde und last not least das negative Ergebniss zahlreicher Untersuchungen auf Trichophytonpilze.

Dass die wenigen lichen-ähnlichen Knötchen eine Rubricirung unseres Falles in die Lichengruppe nicht veranlassen könne, liegt auf der Hand; dazu kommt, dass sie grade während und zwar in den letzten Tagen einer Arsenbehandlung, worauf ich später noch zurückkomme, auftraten.

Während also die obigen Krankheiten aus den erwähnten Gründen mit unserem Falle sehr wenig gemein haben, ist von Jadassohn auf dem 4. Congress der deutschen dermatologischen Gesellschaft ein Fall demonstrirt worden, der zu einem Vergleiche mit unserem Falle Sp. in vielen Punkten auffordert.

Jadassohn's Patient, ein junger Mann, zeigte ein Exanthem, das diffus Stamm und Extremitäten befallen hatte. Die Einzelefflorescenzen ähneln sowohl in der Form, wie in ihrer Entwicklung - denn auch hier machten die einzelnen Efflorescenzen eine Reihe von Metamorphosen durch - den unsrigen. Nach Jadassohn's Bericht boten die Knötchen ihm eine Handhabe, differentialdiagnostisch den Lichen ruber in Betracht zu ziehen, eine Krankheit, die er aber aus verschiedenen Gründen gänzlich von seinem Falle trennt. Solche lichenähnliche Knötchen, wie sie in unserem Falle und zwar erst vor kurzem auftraten, konnte $\mathrm{Jadassohn}$ bei seinem Falle nicht bemerken. Auch das Verbalten der Haut nach Abkratzen der 
Schuppe in Bezug auf das Auftreten einer Blutung war bei seinem Patienten dasselbe. Ferner können wir histologisch unseren Fall mit dem J a d a s s o h n's in eine Linie stellen. J a d a ssohn hat seinen Fall als psoriasiformes und lichenoides Exanthem beschrieben, eine Bezeichnung, zu der wir nach dem Auftreten der Lichenknötchen noch mehr Berechtigung haben. Seinem Vorschlage, das Krankbeitsbild als Dermatitis psoriasiformis nodularis zu bezeichnen, möchten wir uns vorerst nicht anschliessen.

Unserer Ansicht nach genügt es, das Exanthem als psoriasiformes und lichenoides Exanthem zu bezeichnen, bis die Betrachtung ähnlicher Fälle aus ätiologischen oder anatomischen Gründen einen geeigneten Namen bietet.

Wir wollen noch einige Worte bezüglich der Behandlung unseres Falles hinzufügen. Alle äusseren Arzneimittel, die wir anwandten, sowohl indifferente (Zinkpaste), wie differente (eine energische Chrysarobinbehandlung, sowie consequente Darreichung von Theerpräparaten) führten zu keinem Ergebniss. Ebensowenig konnten wir nach einer Anzahl intravenöser Arseninjectionen, zu welcher Therapie wir durch die ausnahmslos günstigen Erfolge bei Psoriasis veranlasst wurden ${ }^{1}$ ) - Pat. erhielt 21 intravenöse Injectionen mit im ganzen $180 \mathrm{Mgr}$. Acid arsenic. - eine auch nur geringe Besserung des Leidens constatiren. Aeusserer Umstände halber mussten wir, obwohl Patient die Arsenikinjectionen gut rertrug, die Cur schon nach 21 Injectionen unterbrechen.

Also in therapeutischer Hinsicht konnten wir ebenso wie $\mathrm{Jadassohn}$ in seinem Falle eine völlige Ergebnisslosigkeit auf Arsenik constatiren. Die Chrysarobinbehandlung, die in unserem Falle keine Besserung veranlasste, hat bei $\mathrm{Jada}$ s ohn's Fall nur einen scheinbaren Erfolg gehabt, da sich Recidive bald wieder einstellter. So veranlasst uns auch diese therapeutische Betrachtung, unser Krankbeitsbild mit Jadassohn's psoriasiformem und lichennidem Exanthem zu identificiren.

1) K. Herxheimer, Ueber Psoriasisbehandlung. Berliner klin. Wochenschrift. 1897. Nr. 35. 